\title{
Optimality Criteria for Fuzzy Pseudo Convex Functions
}

\author{
Sanjaya Kumar Behera ${ }^{1 *}$ and Jyoti Ranjan Nayak ${ }^{2}$
}

Department of Mathematics, Institute of Technical Education and Research Siksha O Anusandhan University, Bhubaneswar-751030,Odisha, India; sanjayeast@yahoo.co.in, jrn_iter@yahoo.co.in

\begin{abstract}
Convex optimization can provide both global as well as local solution; in the case of non convex optimization, it is difficult to get global solution. This paper presents some optimality criteria for the non convex programming problem whose objective function is fuzzy pseudo convex functions.
\end{abstract}

Keywords: Fuzzy Pseudo Convex Functions, Fuzzy Quasi Convex Functions, Non Convex Programming Problem

\section{Introduction}

An optimization problem is convex if it is the minimization of a convex function ${ }^{11}$ (or maximization of a concave function) where the admissible points are in a convex set $^{14}$. The fundamental results in convex analysis ${ }^{6,12}$ say that a locally optimal solution of a convex problem is also globally optimal. If the problem is convex it is enough to guarantee that the solution is globally optimal. In non convex optimization, ${ }^{7}$ choosing the best optimal solution among different local optimal solutions is a difficult task. Convex optimization ${ }^{2,3}$ has provided both a powerful tool and an intriguing mentality to the analysis and design of varieties of models over the last few years. The challenge is on non convex optimization problems $s^{4,16,17}$ and their applications. Deriving the optimality criteria of non-convex programming problem is difficult task in real field. Weir ${ }^{19}$ has derived the optimal solution in non linear programming problem duality with strong pseudo convex function. Rueda et al. ${ }^{13}$ have derived the optimality criteria in mathematical programming with invex function ${ }^{2,3,9,10}$. Sakawa et al..$^{16,17}$ have derived some results on non-convex programming problems in fuzzy domain $^{5,8}$ as well as genetic algorithm. Behera and Nayak ${ }^{18}$ have proposed a method to get the fuzzy optimal solution for non-linear programming problem ${ }^{2,15,18}$. Considering the existing result, we have presented some optimality criteria for non-convex programming problems emphasis on fuzzy pseudo convex functions ${ }^{19}$.

\section{DEFINITION 1.1}

Convex Function: Let $\mathrm{S}$ be a nonempty convex set in $\mathrm{R}^{\mathrm{n}}$. The function $f: S \rightarrow R$ is said to be convex in $\mathrm{S}$ if

$f(\lambda x+(1-\lambda) y) \leq \lambda f(x)+(1-\lambda) f(y)$ for each $x, y \in S$ and for each $\lambda \in[0,1]$.

\section{DEFINITION 1.2}

Concave Function: Let $\mathrm{S}$ be a nonempty convex set in $\mathrm{R}^{\mathrm{n}}$. The Function $f: S \rightarrow R$ is said to be concave is $S$ if $f(\lambda x+(1-\lambda) y) \geq \lambda f(x)+(1-\lambda) f(y)$ for each $x, y \in S$ and for each $\lambda \in[0,1]$.

\section{Definition 1.3}

Pseudo Convex Function: Let $\mathrm{f}$ be a function defined on some open set in $\mathrm{R}^{\mathrm{n}}$ containing $\mathrm{S}$. $\mathrm{f}$ is said to be Pseudo convex at $y \in S$, if it is differentiable at $y$ and satisfies the following conditions,

$$
\nabla f(y) \cdot(x-y) \geq 0 \Rightarrow f(x) \geq f(y)
$$


Note: (a) $f$ is said to be Pseudo convex on $S$ if it is Pseudo Convex at each $x \in S$.

(b) When $\nabla f(y) \cdot(x-y) \leq 0 \Rightarrow f(x) \leq f(y)$ then $\mathrm{f}$ is said to be Pseudo concave function.

\section{DEFINITION 1.4}

Quasi Convex Function: Let $\mathrm{S}$ be convex set in $\mathrm{R}^{\mathrm{n}}$ and $\mathrm{f}$ be a function defined on $\mathrm{S}$ then $\mathrm{f}$ is said to be quasi-convex at $y$ if $f(\lambda x+(1-\lambda) y) \leq \min \{f(x), f(y)\}$ for each $x \in S$ and $0 \leq \lambda \leq 1$ or $\nabla f(y) \cdot(x-y) \leq 0$.

If $\nabla f(y) \cdot(x-y) \geq 0$, then $\mathrm{f}$ is said to be quasi concave.

\section{DEFINITION 1.5}

Invex Function: The function $\mathrm{f}$ is invex at a point $y \in S$ if there exists a function $\eta: S \rightarrow R^{n}$ such that $\mathrm{x}(\mathrm{f})$ such that $f(x)-f(y) \geq \eta(x, y) \cdot \nabla f(y)$ for $x \in S$.

Different types of convex or convex like functions are defined on the fuzzy domain as follows:

Let $\mathrm{K} \subseteq \mathrm{F}_{0}$ where $\mathrm{F}_{0}$ is family of fuzzy numbers.

\section{DEFINITION 1.6}

Fuzzy Convex Function: A fuzzy mapping $F: K \rightarrow F_{0}$ is said to be convex if for every $\lambda \in[0,1]$ and $\tilde{x}, \tilde{y} \in K$.

$$
F(\lambda \tilde{x}+(1-\lambda) \tilde{y}) \leq \lambda F(\tilde{x})+(1-\lambda) F(\tilde{y})
$$

\section{DEFINITION 1.7}

Fuzzy Concave Function: A fuzzy mapping $F: K \rightarrow F_{0}$ is said to be concave if for every $\lambda \in[0,1]$ and $\tilde{x}, \tilde{y} \in K$.

$$
F(\lambda \tilde{x}+(1-\lambda) \tilde{y}) \geq \lambda F(\tilde{x})+(1-\lambda) F(\tilde{y})
$$

\section{DEFINITION 1.8}

Fuzzy Pseudo Convex Function: A fuzzy mapping $F: K \rightarrow F_{0}$ is said to be pseudo convex if $\nabla F(y) .(\tilde{x}-\tilde{y}) \geq 0 \Rightarrow F(\tilde{x}) \geq F(\tilde{y})$ for $x, y \in K$.

\section{DEFINITION 1.9}

Fuzzy Quasi Convex Function: A Fuzzy mapping $F: K \rightarrow F_{0}$ is said to be quasi convex if for every $\lambda \in[0,1]$ and $\tilde{x}, \tilde{y} \in K$.

$F(\lambda \tilde{x}+(1-\lambda) \tilde{y} \leq \operatorname{Sup}\{F(\tilde{x}), F(\tilde{y})\}$ or $\nabla F(\tilde{y}) .(\tilde{x}-\tilde{y}) \leq 0$

\section{DEFINITION 1.10}

Fuzzy Quasi Concave Function: A Fuzzy mapping $F: K \rightarrow F_{0}$ is said to be quasi concave of for each $\lambda \in[0,1]$ and $\tilde{x}, \tilde{y} \in K$.

$$
F(\lambda \tilde{x}+(1-\lambda) \tilde{y} \geq \operatorname{Inf}\{F(\tilde{x}), F(\tilde{y})\}
$$

\section{DEFINITION 1.1 1}

Fuzzy Invex Function: A Fuzzy mapping $F: K \rightarrow F_{0}$ is said to be invex if $F(\tilde{x})-F(\tilde{y}) \geq \eta(\tilde{x}, \tilde{y}) . \nabla F(\tilde{y})$ for $\tilde{x}, \tilde{y} \in K$.

\section{THEOREM 1.12}

Let ' $\mathrm{f}$ ' be differentiable on the open set $\mathrm{C} \subset \mathrm{R}^{\mathrm{n}}$.

(i) If $\mathrm{f}$ is pseudo convex on $\mathrm{C}$ then it is also quasi convex on $\mathrm{C}$ and a global minimum at any $\mathrm{x} \in \mathrm{C}$ such that $\nabla f(x)=0$,

(ii) If $\mathrm{f}$ is quasiconvex on $\mathrm{C}$ and has local minimum at any $\mathrm{x} \in \mathrm{C}$ such that $\nabla f(x)=0$, then it is pseudo convex on $\mathrm{C}$.

Various type of mathematical model of non-convex optimization problem are presented in section-2. Some optimality criteria for those non-convex optimization problems are discussed and verification of the optimality criteria, examples are done with the help of fuzzy arithmetic ${ }^{1,5}$ in section-3. Finally the conclusion is given in Section 4.

\section{Optimization Model}

Fuzzy nonconvex optimization model may be analysed by classifying them in the following manner:

CASE-1: Unconstrained fuzzy nonconvex programming problem:

\section{Minimize $\mathrm{f}(\tilde{x})$}

subject to $\tilde{x} \in \mathrm{K}$

where $\mathrm{f}(\tilde{x})$ is either fuzzy pseudo convex or fuzzy quasi convex functions.

CASE-2 : Constrained fuzzy nonconvex programming problem:

These may be of four types:

$$
\begin{aligned}
\text { minimize } & \left\{\mathrm{f}(\tilde{\mathrm{x}}) / \mathrm{g}_{\mathrm{i}}(\tilde{\mathrm{x}}) \lesssim 0\right\} \\
\text { and } & \tilde{\mathrm{x}} \gtrsim 0
\end{aligned}
$$

where $\mathrm{f}(\tilde{x})$ is fuzzy pseudo convex and $g_{i}(\tilde{x})$ are fuzzy convex.

$$
\begin{array}{cl}
\text { minimize } & \left\{\mathrm{f}(\tilde{\mathrm{x}}) / \mathrm{g}_{\mathrm{i}}(\tilde{\mathrm{x}})<0\right\} \\
\text { and } & \tilde{\mathrm{x}} \gtrsim 0
\end{array}
$$

where $\mathrm{f}(\tilde{x})$ is fuzzy quasi convex and $g_{i}(\tilde{x})$ are fuzzy convex. 
(iii)

$$
\begin{aligned}
\text { minimize } & \left\{\mathrm{f}(\tilde{\mathrm{x}}) / \mathrm{g}_{\mathrm{i}}(\tilde{\mathrm{x}}) \lesssim 0\right\} \\
\text { and } & \tilde{\mathrm{x}} \gtrsim 0
\end{aligned}
$$

where $\mathrm{f}(\tilde{x})$ is fuzzy pseudo convex and $g_{i}(\tilde{x})$ are fuzzy quasi convex.

$\begin{aligned} \text { (iv) } \quad \operatorname{minimize} & \left\{\mathrm{f}(\tilde{\mathrm{x}}) / \mathrm{g}_{\mathrm{i}}(\tilde{\mathrm{x}}) \lesssim 0\right\} \\ \text { and } & \tilde{\mathrm{x}} \gtrsim 0\end{aligned}$

where $\mathrm{f}(\tilde{x})$ is fuzzy convex and $g_{i}(\tilde{x})$ are fuzzy pseudo convex or fuzzy quasi convex.

\section{Optimality Criteria}

In this section, the optimality conditions for all the above fuzzy nonconvex programming problems are discussed.

\section{Case-1:}

(a) Let $\mathrm{f}(\tilde{x})$ be a fuzzy pseudo convex function. Then $\tilde{x}_{0}$ is fuzzy global minimum solution if and only if $\nabla f\left(\tilde{x}_{0}\right) \approx 0$

(b) $\mathrm{f}(\tilde{x})$ is fuzzy quasi convex function at $\tilde{x}_{0}$ and has fuzzy local minimum at $\tilde{x}_{0}$ such that $\nabla f\left(\tilde{x}_{0}\right)=0$, then $\mathrm{f}$ to be fuzzy pseudo convex function satisfying the property as (a).

(i) The necessary conditions that the fuzzy minimum solution exists at $\tilde{x}_{0}$ in $\mathrm{f}(\tilde{x})$ if it satisfy he following conditions;

$$
\begin{aligned}
& \nabla f\left(\tilde{x}_{0}\right)+\lambda \nabla g_{i}\left(\tilde{x}_{0}\right)=0 \\
& \lambda g_{i}\left(\tilde{x}_{0}\right)=0 \\
& \lambda \geq 0
\end{aligned}
$$

The sufficient condition that the $\mathrm{f}(\tilde{x})$ has fuzzy global minimum value at $\tilde{x}_{0}$ if $\mathrm{f}(\tilde{x})$ is fuzzy pseudo convex at $\tilde{x}_{0}$.

\section{ProOF :}

Let $\tilde{x}_{0}$ be the global minimum, then $\nabla f\left(\tilde{x}_{0}\right)=0$

Suppose that $\nabla f\left(\tilde{x}_{0}\right) \approx 0$

$$
\begin{aligned}
& \Rightarrow \nabla f\left(\tilde{x}_{0}\right)\left(\tilde{x}-\tilde{x}_{0}\right)=0 \quad \text { for each } \tilde{x} \in K \\
& \Rightarrow f(\tilde{x}) \gtrsim f\left(\tilde{x}_{0}\right) \text { as } \mathrm{f} \text { is fuzzy pseudo convex } \\
& \Rightarrow \tilde{x}_{0} \text { is the global minimum. }
\end{aligned}
$$

Conversely, let $\tilde{x}_{0}$ is the optimal solution of $f(\tilde{x})$ so $f(\tilde{x}) \gtrsim f\left(\tilde{x}_{0}\right)$

$$
\begin{aligned}
& \Rightarrow f(\tilde{x})-f\left(\tilde{x}_{0}\right) \gtrsim 0 \\
& \Rightarrow \frac{f(\tilde{x})-f\left(\tilde{x}_{0}\right)}{\tilde{x}-\tilde{x}_{0}} \cdot\left(\tilde{x}-\tilde{x}_{0}\right) \gtrsim 0 \\
& \Rightarrow \nabla f\left(\tilde{x}_{0}\right)\left(\tilde{x}-\tilde{x}_{0}\right) \gtrsim 0 \\
& \Rightarrow \nabla f\left(\tilde{x}_{0}\right)\left(\tilde{x}-\tilde{x}_{0}\right)=0 \\
& \Rightarrow \nabla f\left(\tilde{x}_{0}\right)=0
\end{aligned}
$$

(ii) The necessary conditions that the fuzzy minimum solution exists at $\tilde{x}_{0}$ for $\mathrm{f}(\tilde{x})$ if it satisfy the following conditions;

$$
\begin{aligned}
& \nabla f\left(\tilde{x}_{0}\right)+\lambda \nabla g_{i}\left(\tilde{x}_{0}\right)=0 \\
& \lambda g_{i}\left(\tilde{x}_{0}\right)=0 \\
& \lambda \geq 0
\end{aligned}
$$

The sufficient condition that the $\mathrm{f}(\tilde{x})$ has fuzzy global minimum value at $\tilde{x}_{0}$ if $\mathrm{f}(\tilde{x})$ is fuzzy quasi convex at $\tilde{x}_{0}$ and $\nabla f\left(\tilde{x}_{0}\right)=0$.

(iii) The necessary condition that the point $\tilde{x}_{0}$ to be fuzzy minimum solution of $\mathrm{f}(\tilde{x})$ if it satisfy the following necessary conditions,

$$
\begin{aligned}
& \nabla f\left(\tilde{x}_{0}\right)+\lambda \nabla g_{i}\left(\tilde{x}_{0}\right)=0 \\
& \lambda g_{i}\left(\tilde{x}_{0}\right)=0 \\
& g_{i}\left(\tilde{x}_{0}\right) \leq 0 \\
& \lambda \geq 0
\end{aligned}
$$

The sufficient conditions $\mathrm{x}_{0}$ is a fuzzy optimal solution of the minimization problem i.e., $\mathrm{f}\left(\tilde{x}_{0}\right)=\min . \mathrm{f}(\mathrm{x})$ if $\mathrm{f}$ be a fuzzy Pseudo Convex at $\tilde{x}_{0}$ and $g_{i}$ be differentiable and fuzzy quasi convex at $\tilde{x}_{0}$. In case of maximization problem $\mathrm{f}$ is fuzzy Pseudo concave function and $g_{i}$ are differentiable fuzzy quasi convex at $\tilde{x}_{0}$.

\section{Proof :}

$g_{i}(\tilde{x}) \leq 0$ for all $\tilde{x} \in K$ and $\mathrm{i}=1,2,3, \ldots . \mathrm{n}$.

Let $g_{i}(\tilde{x})$ be fuzzy quasi convex on $\mathrm{K}$

$\Rightarrow \nabla g_{i}(\tilde{x})(\tilde{y}-\tilde{x}) \lesssim 0$ for each $\tilde{x}, \tilde{y} \in K$

For any positive value of $\lambda$ we put

$$
\lambda_{i} \nabla g_{i}(\tilde{x})(\tilde{y}-\tilde{x}) \lesssim 0 .
$$

Since $\tilde{x}_{0}$ is the solution,

$\lambda_{i} \nabla g_{i}(\tilde{x})(\tilde{y}-\tilde{x}) \lesssim 0$ for all $\mathrm{i}$

$$
\Rightarrow \lambda \nabla g_{i}(\tilde{x})(\tilde{y}-\tilde{x}) \lesssim 0
$$


But, $\left[\nabla f\left(\tilde{x}_{0}\right)+\lambda \nabla g_{i}\left(\tilde{x}_{0}\right)\right] \approx 0$

$\Rightarrow\left[\nabla f\left(\tilde{x}_{0}\right)+\lambda \nabla g_{i}\left(\tilde{x}_{0}\right)\right]\left(\tilde{y}-\tilde{x}_{0}\right)=0$ for all $\tilde{y} \in K$

$\Rightarrow \nabla f\left(\tilde{x}_{0}\right) \cdot\left(\tilde{y}-\tilde{x}_{0}\right) \lambda \gtrsim 0\left(\because \lambda \nabla g_{i}\left(\tilde{x}_{0}\right)\left(\tilde{y}-\tilde{x}_{0}\right) \lesssim 0\right.$

(Sum is zero one is - ve so other one + ve)

$\Rightarrow \mathrm{f}(\tilde{x})$ is fuzzy Pseudo convex at $\tilde{x}_{0}$.

$\Rightarrow f(\tilde{y}) \geq f\left(\tilde{x}_{0}\right)$ for all $\tilde{y} \in K$

$\Rightarrow f\left(\tilde{x}_{0}\right)=\min _{\tilde{x} \in K} f(\tilde{y})$

So, $\tilde{x}_{0}$ is minimum value of $\mathrm{f}(\tilde{x})$.

(iv) The necessary conditions that the compromise optimal value exists at $\tilde{x}_{0}$ in $\mathrm{f}(\tilde{x})$ if it satisfy he following conditions;

$$
\begin{aligned}
& \nabla f\left(\tilde{x}_{0}\right)+\lambda \nabla g_{i}\left(\tilde{x}_{0}\right)=0 \\
& \lambda g_{i}\left(\tilde{x}_{0}\right)=0 \\
& \lambda \geq 0
\end{aligned}
$$

The sufficient condition that the point $\tilde{x}_{0}$ is compromise local minimum if the Hessian matrix associated with the constraints is negative definite and is local maximum if the Hessian matrix associated with the constraints are positive definite.

\section{EXAMPLE-3.1:}

$$
\begin{array}{ll}
\text { Minimize } & \tilde{x}_{1}^{2}+\tilde{x}_{2}^{2} \\
\text { subject to } & \tilde{x}_{1}+2 \tilde{x}_{2} \leq \tilde{5} \\
& 3 \tilde{x}_{1}+\tilde{x}_{2} \leq \tilde{4} \\
\text { and } & \tilde{x}_{1}, \tilde{x}_{2} \geq 0
\end{array}
$$

To find $x=\left(\tilde{x}_{1}, \tilde{x}_{2}\right)$ which minimize $\mathrm{f}(\mathrm{x})=\tilde{x}_{1}^{2}+\tilde{x}_{2}^{2}$ and satisfies the constraints.

$$
\begin{array}{ll} 
& \tilde{x}_{1}+2 \tilde{x}_{2} \leq \tilde{5} \\
& 3 \tilde{x}_{1}+\tilde{x}_{2} \leq \tilde{4} \\
\text { and } \quad & \tilde{x}_{1}, \tilde{x}_{2} \geq 0
\end{array}
$$

By using the necessary conditions:

(i) $\nabla f(x)+\lambda \nabla g(x) \approx 0$

$$
\begin{aligned}
& \left(\frac{\partial f}{\partial \tilde{x}_{1}}, \frac{\partial f}{\partial \tilde{x}_{2}}\right)-\tilde{\lambda}_{1}\left(\frac{\partial g_{1}}{\partial \tilde{x}_{1}}, \frac{\partial g_{2}}{\partial \tilde{x}_{2}}\right)-\tilde{\lambda}_{2}\left(\frac{\partial g_{2}}{\partial \tilde{x}_{1}}, \frac{\partial g_{2}}{\partial \tilde{x}_{2}}\right)=0 \\
& \Rightarrow\left(2 \tilde{x}_{1}, 2 \tilde{x}_{2}\right)-\tilde{\lambda}_{1}(1,2)-\tilde{\lambda}_{2}(3,+1)=0 \\
& \Rightarrow 2 \tilde{x}_{1}-\tilde{\lambda}_{1}-3 \tilde{\lambda}_{2}=0 \\
& 2 \tilde{x}_{1}-2 \tilde{\lambda}_{1}-\tilde{\lambda}_{2}=0
\end{aligned}
$$

(ii) $\lambda_{i} g_{i}(x)=0$

$$
\begin{aligned}
& \tilde{\lambda}_{1}\left(\tilde{x}_{1}+2 \tilde{x}_{2}-\tilde{5}\right)=0 \\
& \tilde{\lambda}_{2}\left(3 \tilde{x}_{1}+\tilde{x}_{2}-\tilde{4}\right)=0
\end{aligned}
$$

(iii) $\tilde{\lambda}_{i} \geq 0$

Solving Equation (1),(2),(3),(4) by using $\tilde{x}_{1}=\left(\underline{x_{1}}, x_{1}, \bar{x}_{1}\right), \tilde{x}_{2}=\left(\underline{x_{2}}, x_{2}, \bar{x}_{2}\right)$,

$\tilde{\lambda}_{1}=\left(\underline{\lambda_{1}}, \lambda_{1}, \tilde{\lambda}_{1}\right), \tilde{\lambda}_{2}=\left(\underline{\lambda_{2}}, \lambda_{2}, \tilde{\lambda}_{2}, \tilde{5}=(4,5,6)\right.$ and $\tilde{4}=(2,4,5)$ we get,

$$
\begin{aligned}
x_{0}: \quad \tilde{x}_{1} & =\left(0, \frac{3}{5}, \frac{4}{5}\right) \\
\tilde{x}_{2} & =\left(2, \frac{11}{5}, \frac{13}{5}\right) \\
\tilde{\lambda}_{1} & =\left(-\frac{4}{5}, \frac{-2}{5}, \frac{-2}{5}\right) \\
\tilde{\lambda}_{2} & =\left(\frac{12}{5}, \frac{12}{5}, \frac{14}{5}\right)
\end{aligned}
$$

Fuzzy optimal value of $\mathrm{f}(\mathrm{x})$ is $\left(4, \frac{130}{25}, \frac{185}{25}\right)$.
$\quad$ Now $\nabla f\left(\tilde{x}_{0}\right) \cdot\left(x-\tilde{x}_{0}\right)$

$$
\begin{aligned}
= & {\left[\left(0, \frac{6}{5}, \frac{8}{5}\right),\left(4, \frac{22}{5}, \frac{26}{5}\right)\right] \cdot\left[\left(x, x-\frac{3}{5}, \bar{x}-\frac{4}{5}\right)\right.} \\
& \left.\left(\underline{y}-2, y-\frac{11}{5}, \bar{y}-\frac{13}{5}\right)\right] \\
\Rightarrow & \nabla f\left(\tilde{x}_{0}\right) \cdot\left(x-\tilde{x}_{0}\right) \geq 0 \\
\Rightarrow & x>\tilde{x}_{0} \\
\Rightarrow & f(x) \gtrsim f\left(\tilde{x}_{0}\right)
\end{aligned}
$$

So $\mathrm{f}(\mathrm{x})$ is Pseudo Convex at $\tilde{x}_{0}$ As $\mathrm{g}_{\mathrm{i}}(\mathrm{x})$ are linear, they are fuzzy convex so they are fuzzy quasiconvex.

Hence $\tilde{x}_{0}$ is the compromise minimum optimal value.

\section{EXAMPLE-3.2:}

Minimize $\tilde{x}^{3}+\tilde{x}$

Subject to $\tilde{x}^{2} \leq \tilde{2}$ and $\tilde{x} \geq \tilde{\sim}$

By using the necessary conditions:

(i) $\nabla f(\tilde{x})+\lambda \nabla g(\tilde{x})=0$

$$
\Rightarrow \frac{\partial f}{\partial \tilde{x}}-\tilde{\lambda} \frac{\partial g}{\partial \tilde{x}} \approx 0
$$




$$
3 \tilde{x}^{2}+1-2 \tilde{\lambda} \tilde{x}=0
$$

(ii) $\tilde{\lambda} g_{1}(\tilde{x})=0$

$$
\begin{gathered}
\tilde{x}^{2}-\tilde{2}=0 \\
\tilde{\lambda} \gtrsim 0
\end{gathered}
$$

Solving (5), (6), (7) by using $\tilde{x}=(\underline{x}, x, \bar{x}), \tilde{\lambda}=(\underline{\lambda}, \lambda, \bar{\lambda})$ and $\tilde{4}=(3,4,6)$ we get;

$$
\begin{aligned}
\tilde{x} & =(\sqrt{3}, 2, \sqrt{6}) \\
\tilde{\lambda} & =\left(\frac{5}{\sqrt{3}}, \frac{13}{4}, \frac{19}{2 \sqrt{6}}\right)
\end{aligned}
$$

Now $\nabla f(\tilde{x}) \cdot(\tilde{y}-\tilde{x})$

$$
\begin{aligned}
& \Rightarrow \quad\left(3 \tilde{x}^{2}+1\right)(\underline{y}-\sqrt{3}, y-2, \bar{y}-\sqrt{6}) \geq 0 \\
& \Rightarrow \quad(10,13,19)(\underline{y}-\sqrt{3}, y-2, \bar{y}-\sqrt{6}) \geq 0 \\
& \Rightarrow \quad \underline{y} \geq \sqrt{3}, y \geq 2, \bar{y} \geq \sqrt{6}
\end{aligned}
$$

$\Rightarrow f(\tilde{y}) \gtrsim f(\tilde{x})$ for all $\tilde{y}$ (since $\mathrm{f}$ is increasing function) $\Rightarrow \mathrm{f}$ is pseudo convex function

But $\mathrm{g}(\mathrm{x})$ is a convex function so it is a quasi convex. Hence the fuzzy minimum solution exist and that is

$$
(\sqrt{3}, 2, \sqrt{6})^{3}+(\sqrt{3}, 2, \sqrt{6})=(4 \sqrt{3}, 10,7 \sqrt{6})
$$

\section{Conclusion}

Non convex optimization problems in fuzzy domain have been considered. The objective functions are pseudo convex and the constraints are quasi convex functions. The decision variables are assumed to be fuzzy triangular numbers. The class of pseudo and quasi convex functions are more general in nature in comparision with usual convex functions. The fuzzy optimal solutions are obtained in a real interval.

\section{References}

1. Kaufmann A, Gupta MM. Introduction to Fuzzy Arithmetic Theory and Applications. Van Nostrand, Reinhold, New York; 1985.
2. Bazaraa MS, Shetty CM, Nonlinear Programming Theory and Algorithms. Wiley Publishers, New York; 1979 .

3. Bereanu B. Quasi-convexity, strictly quasi-convexity and pseudo convexity of composition objective functions. Review of Francaise Automat. Informat. Recherche Operationelle. 1972; 6(R-1):15-26.

4. Weir T. On strong pseudo-convexity in non-linear programming duality. Opsearch. 1990; 27:117-27 .

5. Zimmerman HJ. Fuzzy programming and Linear programming with several objective functions. Fuzzy Sets and Systems. 1978; 1:45-55.

6. Taha HA. Introduction to Operations Research, seventh ed., University of Arkansas, Fayetteville; 2005.

7. Nayak JR. Some problems of non-convex programming and the properties of some Non-convex functions. [Ph. D. Thesis], Utkal University; 2004.

8. Zadeh LA. Fuzzy sets. Information and Control. 1965; 8:338-53.

9. Pini R. Invexity and generalized convexity. Optimization. 1991; 21:513-25.

10. Alfio P. Generalized convexity and invexity in Optimization Theory. Appl Math Sci. 2009; 3( 47):2311-25 .

11. Robert AW, Verberg DE. Convex Functions. Academic Press, New York; 1973.

12. Rockafullar RT. Convex Analysis. Princeton University Press, N.J.; 1969.

13. Rueda NG, Hanson MA. Optimality criteria in mathematical programming involving generalized invexity. Math Anal Appl. 1988; 130:375-85.

14. Valentine FA. Convex Sets. Mc. Graw Hill, New York; 1964.

15. Nasseri SH. Fuzzy nonlinear optimization. J Nonlinear Sci Appl. 2008; 1(4):230-35 .

16. Sakawa M, Yauchi K. An interactive fuzzy satisfying method for multiobjective non convex programming problems through floating point genetic algorithms. Cent Eur J Oper Res. 1999; 117(1):113-24.

17. Sakawa M, Yauchi K. Interactive decision making for multiobjective non convex programming problems with fuzzy numbers through coevolutionary genetic algorithms. Fuzzy Sets and Systems. 2000; 114(1):151-65.

18. Behera SK, Nayak RJ. Solution of fuzzy non-linear programming problems with linear constraints. International Journal of Advances in Science and Technology. 2012; $4(2): 43-52$. 